\title{
Association between genetic polymorphism and levothyroxine bioavailability in hypothyroid patients
}

\author{
Merve Arici' ${ }^{1)}$, Ezgi Oztas ${ }^{1)}$, Fatih Yanar $^{2)}$, Nihat Aksakal ${ }^{2)}$, Beyza Ozcinar²) and Gul Ozhan ${ }^{1)}$ \\ 1) Department of Pharmaceutical Toxicology, Faculty of Pharmacy, Istanbul University, Istanbul, Turkey \\ ${ }^{2)}$ Department of General Surgery, Faculty of Medicine, Istanbul University, Istanbul, Turkey
}

\begin{abstract}
Thyroid hormones play a vital role in the human body for growth and differentiation, regulation of energy metabolism, and physiological function. Hypothyroidism is a common endocrine disorder, which generally results from diminished normal circulating concentrations of serum thyroxine (fT4) and triiodothyronine (fT3). The primary choice in hypothyroidism treatment is oral administration of levothyroxine (L-T4), a synthetic T4 hormone, as approximately 100-125 $\mu \mathrm{g} /$ day. Generally, dose adjustment is made by trial and error approach. However, there are several factors which might influence bioavailability of L-T4 treatment. Genetic background could be an important factor in hypothyroid patients as well as age, gender, concurrent medications and patient compliance. The concentration of thyroid hormones in tissue is regulated by both deiodinases enzyme and thyroid hormone transporters. In the present study, it was aimed to evaluate the effects of genetic differences in the proteins and enzymes (DIO1, DIO2, TSHR, THR and UGT) which are efficient in thyroid hormone metabolism and bioavailability of L-T4 in Turkish population. According to our findings, rs225014 and rs225015 variants in $\mathrm{DIO}$, which catalyses the conversion of thyroxine (pro-hormone) to the active thyroid hormone, were associated with TSH levels. It should be given lower dose to the patients with rs225014 TT and rs225015 GG genotypes in order to provide proper treatment with higher effectivity and lower toxicity.
\end{abstract}

Key words: Levothyroxine, Hypothyroid, Genetic polymorphism

THYROID HORMONE-RELATED CLINICAL SYMPTOMS AND LONG-TERM COMPLICATIONS are mainly skin manifestations, atherosclerosis, bone mineral density, heart rate, obesity, hyperlipidaemia, bradycardia, fatigue and depression, which all have serious impact on life quality $[1,2]$. Hypothyroidism, one of the most common endocrine disorders, is caused by lack of action of thyroid hormones that play a vital role in the human body for growth and differentiation, and regulation of energy metabolism, and physiological function. Hypothyroidism generally results from diminished normal circulating concentrations of fT3 and fT4 [3, 4]. The production of thyroid hormones is regulated by hypothalamuspituitary-thyroid axis through a negative feedback system. The secretion of T3 and T4 is controlled by both hypo-

Submitted May. 4, 2017; Accepted Nov. 24, 2017 as EJ17-0162

Released online in J-STAGE as advance publication Jan. 11, 2018

Correspondence to: Prof. Dr. Gul Ozhan, Department of Pharmaceutical Toxicology, Faculty of Pharmacy, Istanbul University, Beyazit 34116, Istanbul, Turkey.

E-mail: gulozhan@istanbul.edu.tr thalamic thyrotropin-releasing hormone, which its action exerts by binding to thyroid hormone receptors (THR $\alpha$ and $\beta$ ) and thyroid stimulating hormone (TSH). TSH exerts its role through binding to TSH receptor (TSHR) $[4,5]$.

L-T4 is a synthetic T4 hormone in the treatment of hypothyroidism. In adults, L-T4 is administered orally as average $1.7 \mu \mathrm{g} / \mathrm{kg}$ body weight/day, being equivalent to approximately $100-125 \mu \mathrm{g}$ per day. It is needed to higher doses than $1.7 \mu \mathrm{g} / \mathrm{kg} /$ day for new-borns, infants and adolescents. Age, gender, concurrent medications, patient compliance, genetic background etc. can influence proper L-T4 treatment $[1,6]$.

In recent years, it is clinically important approach the personalised medicine instead of empirical treatment to enhancing responses among patients [7]. The concentration of thyroid hormones in tissues is regulated by deiodinases and thyroid hormone transporters. Deiodinase enzyme isoforms (DIO1, DIO2, and DIO3) mainly control intracellular thyroid hormone concentrations. DIO enzymes convert biologically active hormone (T3) from 
L-T4. DIO1 and DIO2 catalyze activation of thyroid hormone secretion in contrast to DIO3 playing role inactivation of the secretion. Activities of DIO1 and DIO2 play pivotal role in the negative feedback regulation of pituitary TSH secretion $[8,9]$. UDP-glucuronosyltransferases (UGTs) are responsible for T4 metabolism in human liver as thyroxine glucuronide. Two common UGT1A subfamily enzymes, UGT1A1 and UGT1A3, provide T4 glucuronidation in humans [10]. It was determined different responses dependently of genetic factors in the L$\mathrm{T} 4$ treatment. The previously studies reported the genetic variations on proteins and enzymes have been played role in thyroid hormones metabolism, serum thyroid hormone concentrations, and bioavailability of L-T4 $[8,11]$. Therefore, we aimed to comprehensively evaluate the effect of genetic differences in the proteins and enzymes (DIO1, DIO2, TSHR, THR $\alpha$ and UGT) which are efficient in thyroid hormones metabolism on bioavailability of L-T4.

\section{Material and Methods}

Sample collection: This is a cross-sectional study approved by ethical committee of Istanbul University Istanbul Medical Faculty (2015/740), and was carried out in accordance with the Helsinki Declaration of 1975, with all amendments and revisions. A total of 94 unrelated patients with secondary hypothyroidism due to total thyroidectomy were recruited from Endocrine Surgery Clinic of General Surgery Department, Istanbul Medical Faculty, Istanbul University between March 2015 and October 2016. Patients included in this study are all those who completed puberty, aged with $18-75$, underwent total thyroidectomy for different causes (multi nodular goitre, suspicion of cancer, pressure effect of large goitre and susbternal goitre) and on two types of doses of L-T4 as high and low doses $(<1.7 \mu \mathrm{g} / \mathrm{kg} /$ day and $\geq 1.7$ $\mu \mathrm{g} / \mathrm{kg} / \mathrm{day}$ ). In the studied group, L-T4 was administered as a single dose daily. Neoplasm, liver dysfunction, renal failure, and psychiatric condition not related to hypothyroidism symptoms. Also, pregnancy and alcohol abuse patients on L-T4 treatment were accepted as the excluded criteria. TSH, fT3 and fT4 levels were measured by GenWay Biotach Inc. (San Diego, CA, USA) colorimetric ELISA kits according to manufacturer's instructions by specialists in biochemistry laboratories of Istanbul Medical Faculty.

Genotyping: DNA was isolated from venous blood samples by High Pure PCR Template Preparation Kit
(Roche, Germany). The single nucleotide polymorphisms (SNPs) analysis was performed on real-time PCR platform (LightCycler 480, Roche, Germany) using LightCycler FastStart DNA Master HybProbe and Roche LightSNP assay probes (Roche, Germany). The studied SNPs were rs11206244, rs2235544 in DIO1; rs225014, rs225015, rs12885300 in DIO2; rs939348 in THRa; rs4903957, rs1991517, rs2239610, rs2268458 in TSHR; rs1983023, rs3806596 in UGT1A3 and rs8175347 in UGT1A1*28.

Statistical analysis: All statistical analyses were performed using Statistical Package for Social Sciences (SPSS) software (Version 20, Chicago, USA). The HardyWeinberg Equilibrium (HWE) analysis was performed to compare the observed and expected genotype frequencies of subject by using the chi-square $\left(\chi^{2}\right)$ test. It was confirmed that the studied population was randomized by the results obtained from HWE analysis. Sample size was calculated with $95 \%$ confidence, and a margin of error 5\% for an assuming population proportion of 0.5 , and unlimited population size. Continuous variables were expressed as mean \pm standard deviation (SD) whereas discrete variables were expressed as frequencies. The differences were accomplished by comparison with one way ANOVA. Post Hoc Tukey test and Independent $t$ Test was applied to evaluate for the association between the clinical and biochemical characteristics and the studied genes. A significant difference is considered at $p<$ 0.05 .

\section{Results}

It was evaluated the effects of 13 SNPs of DIO1, DIO2, THR, TSHR and UGTIA on thyroid hormones metabolism and bioavailability of L-T4. The mean age of patients was $51.35( \pm 1.53)$ years, the mean body mass index (BMI, $\left.\mathrm{kg} / \mathrm{m}^{2}\right)$ was $29.16( \pm 0.65)$ and of the all participants $82.61 \%$ were female and $48.89 \%$ have familial background of thyroid disorders. Of the all participants, $22.83 \%$ were dyslipidemic, $23.92 \%$ were diabetic, and $20.41 \%$ were hypertensive. The patients were also evaluated by dividing into two groups based on current replacement L-T4 dose: low dose group $(<1.7 \mu \mathrm{g} / \mathrm{kg} /$ day $)$ and high dose group $(\geq 1.7 \mu \mathrm{g} / \mathrm{kg} /$ day $)$. Age distribution between two groups did not show any difference. As it is expected, the hypothyroid patients who received high dose of LT-4 had a slightly lower BMI. Also, it was found that high dose of LT-4 treatment was associated with lower levels of TSH, and higher levels of fT3 and 
Table 1 Characteristics of hypothyroid patients based on the dose of LT-4 replacement

\begin{tabular}{lccc}
\hline \multicolumn{1}{c}{ Variable } & Low dose $<1.7 \mu \mathrm{g} / \mathrm{kg} /$ day $(n=66)$ & High dose $\geq 1.7 \mu \mathrm{g} / \mathrm{kg} /$ day $(n=28)$ & $p$ value \\
\hline Age & $52.00 \pm 4.06$ & $45.08 \pm 2.03$ & 0.101 \\
BMI $\left(\mathrm{kg} / \mathrm{m}^{2}\right)$ & $29.82 \pm 0.81$ & $26.99 \pm 1.41$ & 0.079 \\
TSH $(\mathrm{mIU} / \mathrm{L})$ & $1.93 \pm 0.37$ & $0.51 \pm 0.29$ & $\mathbf{0 . 0 2 9}$ \\
fT3 $(\mathrm{pmol} / \mathrm{L})$ & $4.71 \pm 0.16$ & $5.39 \pm 0.27$ & $\mathbf{0 . 0 4 8}$ \\
fT4 $(\mathrm{pmol} / \mathrm{L})$ & $19.73 \pm 0.66$ & $22.09 \pm 0.75$ & $\mathbf{0 . 0 4 5}$ \\
\hline
\end{tabular}

Bold values mean $p<0.05$

fT4 $(p<0.05)$ (Table 1). The typical reference ranges for thyroid function parameters are between $0.4-4 \mathrm{mIU} / \mathrm{L}$ for TSH, 3.5-7.8 pmol/L for $\mathrm{fT} 3$, and $9-25 \mathrm{pmol} / \mathrm{L}$ for fT4 [12]. Similarly, the mean values of the parameters were $1.54 \pm 0.44 \mu \mathrm{g} / \mathrm{kg} /$ day for L-T4 daily dose, 1.79 $\pm 2.33 \mathrm{mIU} / \mathrm{L}$ for TSH, $4.69 \pm 1.02 \mathrm{pmol} / \mathrm{L}$ for fT3, and $19.75 \pm 4.22 \mathrm{pmol} / \mathrm{L}$ for fT 4 in the studied group.

Genotype distributions of studied SNPs are shown in Table 2. All genotypes were found to be consistent with the HWE $(p>0.05)$. Heterozygous type $[A T /-]$ for UGT1A1*28 (rs8175347) was not observed. Interestingly, we observed that DIO2 rs225015 G and TSHR rs $1991517 C$ alleles were wild types with the $66.1 \%$ and $88.9 \%$ frequencies, respectively. However, the ancestral alleles were indicated as $A$ and $G$ according to NCBI SNP database [https://www.ncbi.nlm.nih.gov/projects/SNP].

There was no significant correlation among genotypes and L-T4 dose $(p>0.05)$, the biochemical parameters including thyroid function test (TSH, fT3 and fT4), and body mass index. A statistical significance was found between DIO2 rs225014 and TSH levels. Homozygous wild type (TT, Thr/Thr) for DIO2 rs225014 was associated with higher levels of TSH $(p<0.05)$. Besides, DIO2 rs225015 $G G$ genotype was found to be associated with higher levels of TSH compared with the $A A$ genotype ( $p$ $<0.05)$. Homozygous mutant types of TSHR rs4903957 $(A A)$, rs1991517 (CC), rs2239610 (CC) and rs2268458 (CC) were related with higher levels of TSH. Although obtained significant differences in the values, the association did not reach statistical significance. Probably the reason is their excessive SDs. Additionally, homozygous variant types of THR $\alpha$ rs939348 (TT) and UGT1A3 rs1983023 (CC) were related with lower levels of TSH (Table 3).

\section{Discussion}

The present study is a comprehensive cross-sectional study, and first study to evaluation the genetic profiles of Turkish people in the genes related to thyroid metabolism (Table 3). There are limited publications about the relationship between the gene variants and drug-response in hypothyroidism treatment $[1,2,8,10,13-20]$.

Panicker et al. [14] suggested that DIO1 rs2235544 has a correlation with circulating fT3/fT4 levels both in the population with thyroid hormone replacement and in the general population. Rs2235544 $C$ allele was associated with increased fT3 and decreased fT4 levels, however not associated with serum TSH levels. They also reported no association between DIO1 rs11206244, which increase enzyme activity, and the levels of mentioned serum parameters. In the previous studies, it was suggested that the patient with carrying $C$ allele of rs2235544 should be treated higher doses of L-T4 because $C$ allele increases the function of DIOI [17, 18]. However, Santoro et al. [10] stated no association between dose and these genes. Similar to Santoro et al. [10], in the present study, we observed that the values of TSH, fT3 and fT4 were in normal range in patients with variant and wild alleles of DIOI rs11206244 and rs2233544. There was no association between DIO1 (rs2233544) genotype and the TSH, fT3 and fT4 levels.

Heemestra et al. [19] observed no differences between DIO2 rs225014 and thyroid hormone levels and L-T4 doses adjusted for age, gender, BMI and serum TSH levels. Similar results were observed by Panicker et al. [14] and Al-Azzam et al. [8]. However, Torlontano et al. [15] stated that the patient with homozygous variant type (CC, Ala/Ala) for DIO2 rs225014 needed the higher dose of L-T4 to provide favourable TSH levels. They reported that an approximate $20 \%$ higher dose was needed in patient with homozygous variant type for target TSH levels to be reached. And, they suggested a reduced pituitary feedback due to abnormal DIO2 hypothalamic/ pituitary activity. As it is known, DIO2 activity plays an important role in the negative feedback regulation of 
Table 2 The frequencies of 13 gene variants in the patients with hypothyroidism

\begin{tabular}{|c|c|c|c|c|}
\hline \multicolumn{2}{|c|}{ SNP } & \multicolumn{3}{|c|}{$\begin{array}{c}\text { Genotype } \\
n(\%)\end{array}$} \\
\hline \multirow{4}{*}{ DIOI } & \multirow{2}{*}{ rs 11206244} & $\mathrm{CC}$ & $\mathrm{CT}$ & $\mathrm{TT}$ \\
\hline & & $47(52.2)$ & 35 (38.9) & $8(8.9)$ \\
\hline & \multirow{2}{*}{ rs 2235544} & $\mathrm{CC}$ & $\mathrm{CA}$ & AA \\
\hline & & $33(36.7)$ & $44(48.9)$ & $13(14.4)$ \\
\hline \multirow{6}{*}{$\mathrm{DIO} 2$} & \multirow{2}{*}{ rs225014 } & $\mathrm{TT}$ & $\mathrm{TC}$ & $\mathrm{CC}$ \\
\hline & & $38(45.2)$ & $34(40.5)$ & $12(14.3)$ \\
\hline & \multirow{2}{*}{ rs225015 } & AA & AG & GG \\
\hline & & $13(14.4)$ & 35 (38.9) & $42(46.7)$ \\
\hline & \multirow{2}{*}{ rs 12885300} & $\mathrm{CC}$ & CT & $\mathrm{TT}$ \\
\hline & & $39(43.8)$ & $43(48.3)$ & 7 (7.9) \\
\hline \multirow{2}{*}{$T H R \alpha$} & \multirow{2}{*}{ rs939348 } & $\mathrm{CC}$ & $\mathrm{CT}$ & $\mathrm{TT}$ \\
\hline & & $49(54.4)$ & 35 (38.9) & $6(6.7)$ \\
\hline \multirow{8}{*}{ TSHR } & \multirow{2}{*}{ rs4903957 } & GG & GA & AA \\
\hline & & $50(55.6)$ & $36(40)$ & $4(4.4)$ \\
\hline & \multirow{2}{*}{ rs1991517 } & GG & GC & $\mathrm{CC}$ \\
\hline & & $3(3.3)$ & $14(15.6)$ & $73(81.1)$ \\
\hline & \multirow{2}{*}{ rs 2239610} & GG & $\mathrm{GC}$ & $\mathrm{CC}$ \\
\hline & & $56(62.2)$ & $32(35.6)$ & $2(2.2)$ \\
\hline & \multirow{2}{*}{ rs 2268458} & $\mathrm{TT}$ & $\mathrm{TC}$ & $\mathrm{CC}$ \\
\hline & & $56(62.2)$ & $32(35.6)$ & $2(2.2)$ \\
\hline \multirow{4}{*}{ UGT1A3 } & \multirow{2}{*}{ rs 1983023} & TT & $\mathrm{TC}$ & $\mathrm{CC}$ \\
\hline & & $41(45.6)$ & $37(41.1)$ & $12(13.3)$ \\
\hline & \multirow{2}{*}{ rs 3806596} & AA & AG & GG \\
\hline & & $28(31.1)$ & $41(45.6)$ & $21(23.3)$ \\
\hline \multirow{2}{*}{$U G T 1 A 1 * 28$} & \multirow{2}{*}{ rs 8175347} & [AT] & {$[\mathrm{AT} /-]$} & {$[-]$} \\
\hline & & $24(26.7)$ & $0(0)$ & $66(73.3)$ \\
\hline
\end{tabular}

pituitary TSH secretion [15]. Similarly, in the present study, a statistical significance was found between $\mathrm{DIO} 2$ rs225014 and TSH levels; homozygous wild type (TT, $T h r / T h r)$ was associated with higher levels of TSH $(p<$ 0.05). Also, DIO2 rs225015 GG genotype was found to be associated with higher levels of TSH compared with the $A A$ genotype $(p<0.05)$. For DIO2 rs225014 and rs225015, their effected genotypes were observed in different frequencies. The common homozygous type for DIO2 rs225014 (TT) and the mutant homozygous types for rs225015 $(G G)$ were associated with higher levels of TSH compared with their other types. Therefore, the patients with for DIO2 rs225014 (TT) and rs225015 $(G G)$ variants have higher TSH levels, and should take higher L-T4 dose.

THR $\alpha$ rs939348 was associated with L-T4 replacement doses [1]. It was suggested that $T$ allele carrying patients should be taken more doses of L-T4 because of the increased enzyme function. Similarly, we observed that the TSH level was lower in the patient with THR $\alpha$ rs939348 TT genotype in same dose L-T4 treatment.

The previous studies stated there was no association between TSHR and L-T4 dose $[20,21]$. In the present study, it was found that homozygous mutant type of 
Table 3 The biochemical characteristics of the DIO1, DIO2, THR , TSHR, UGT1A3 and UGT1A1*28 genotypes of the hypothyroid patients

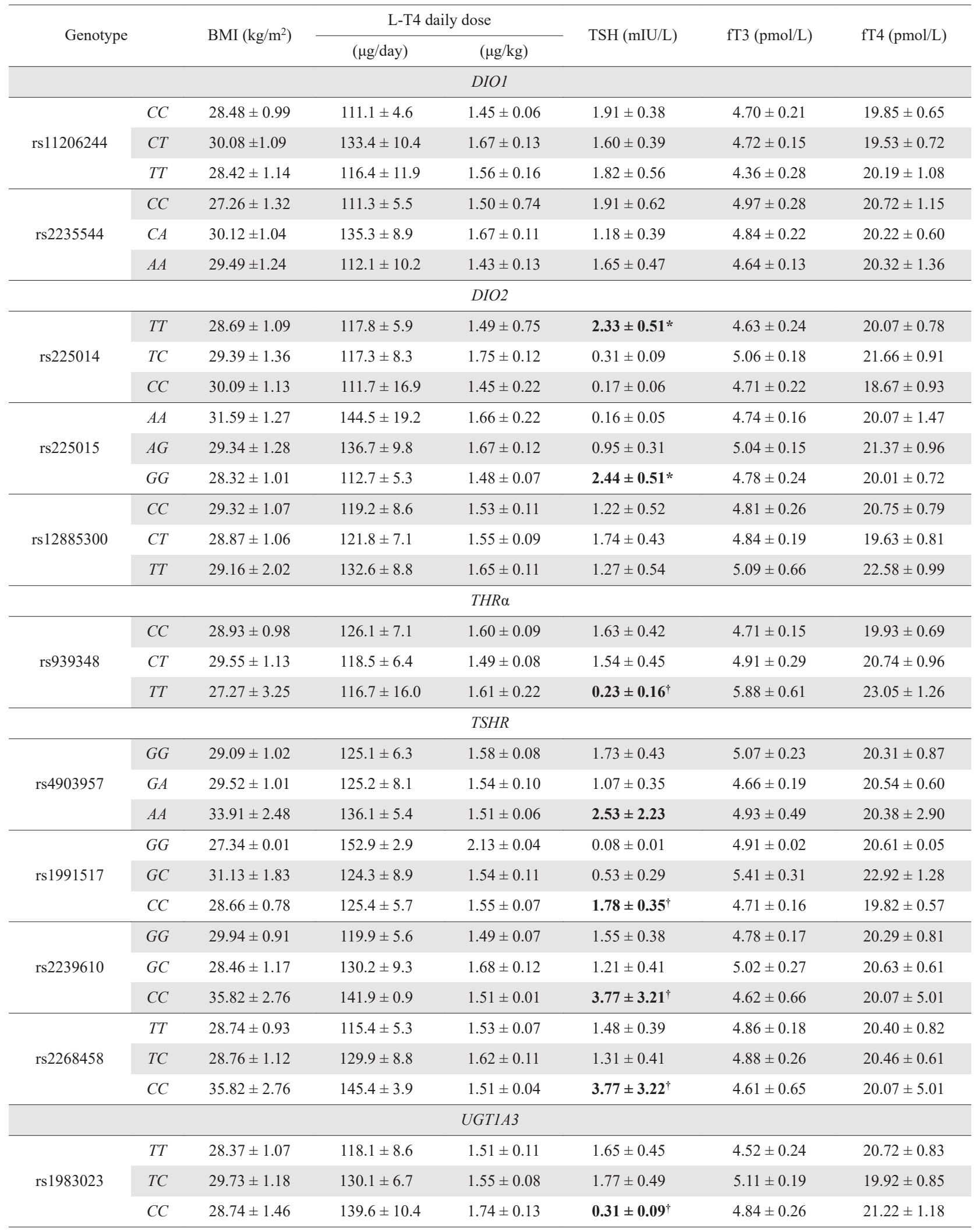


Table 3 Continued

\begin{tabular}{|c|c|c|c|c|c|c|c|}
\hline \multirow{2}{*}{\multicolumn{2}{|c|}{ Genotype }} & \multirow{3}{*}{$\begin{array}{l}\text { BMI }\left(\mathrm{kg} / \mathrm{m}^{2}\right) \\
29.22 \pm 1.15\end{array}$} & \multicolumn{2}{|c|}{ L-T4 daily dose } & \multirow{3}{*}{$\begin{array}{c}\mathrm{TSH}(\mathrm{mIU} / \mathrm{L}) \\
1.82 \pm 0.57\end{array}$} & \multirow{3}{*}{$\begin{array}{c}\mathrm{fT} 3(\mathrm{pmol} / \mathrm{L}) \\
4.58 \pm 0.37\end{array}$} & \multirow{3}{*}{$\begin{array}{l}\mathrm{fT} 4(\mathrm{pmol} / \mathrm{L}) \\
20.47 \pm 0.88\end{array}$} \\
\hline & & & \multirow{2}{*}{$\begin{array}{c}(\mu \mathrm{g} / \text { day }) \\
103.8 \pm 4.7\end{array}$} & \multirow{2}{*}{$\begin{array}{c}(\mu \mathrm{g} / \mathrm{kg}) \\
1.32 \pm 0.06\end{array}$} & & & \\
\hline \multirow{3}{*}{ rs 3806596} & $A A$ & & & & & & \\
\hline & $A G$ & $28.84 \pm 1.14$ & $128.0 \pm 8.6$ & $1.63 \pm 0.11$ & $1.63 \pm 0.51$ & $5.02 \pm 0.19$ & $20.41 \pm 0.90$ \\
\hline & $G G$ & $29.25 \pm 1.41$ & $126.0 \pm 8.5$ & $1.64 \pm 0.11$ & $1.06 \pm 0.41$ & $4.81 \pm 0.22$ & $20.37 \pm 0.92$ \\
\hline \multicolumn{8}{|c|}{$U G T 1 A 1 * 28$} \\
\hline \multirow{3}{*}{ rs8175347 } & {$[A T]$} & $30.11 \pm 1.31$ & $126.3 \pm 9.0$ & $1.54 \pm 0.11$ & $1.07 \pm 0.31$ & $4.85 \pm 0.15$ & $19.41 \pm 0.98$ \\
\hline & {$[A T /-]$} & - & - & - & - & - & - \\
\hline & {$[-]$} & $28.46 \pm 0.83$ & $120.3 \pm 6.1$ & $1.57 \pm 0.08$ & $1.75 \pm 0.41$ & $4.85 \pm 0.19$ & $20.91 \pm 0.63$ \\
\hline
\end{tabular}

* Indicates the statistically significant difference $(p<0.05)$.

${ }^{\dagger}$ Indicates the association that did not reach statistical significance.

The biochemical characteristics for the patients with the DIO1, DIO2, THR, TSHR and UGT1A3 genotypes were analysed with Post Hoc Tukey test. Only, $U G T 1 A 1 * 28$ was analysed with independent- $t$ test because of the absence of heterozygous type.

TSHR rs4903957 (AA), rs1991517 (CC), rs2239610 (CC) and rs2268458 (CC) were related with higher levels of TSH. However, the association did not reach statistical significance probably due to their excessive SDs.

The previous studies have showed a direct or indirect correlation between L-T4 dose and UGTIA. It was also indicated the importance of glucuronidation in $\mathrm{T} 4$ homeostasis and UGT1A1 and UGT1A3. UGT1A1 had a higher affinity than UGTIA3 for T4 in T4 glucuronication while UGTIA3 had higher capacity for T4 glucuronidation. It was stated a significant correlation between UGT1A1*28 and T4 glucuronidation [13]. Graber et al. [13] pointed out hypothyroid patients with homozygous UGT1A1*28 variant should receive a lower L-T4 dose. Santoro et al. [10] and Vargens et al. [16] reported that polymorphic individuals of UGTIA should be received lower doses of L-T4 to reach appropriate levels of TSH due to lower expressions of related SNPs of UGT1A. In our findings, homozygous variant type (CC) of UGT1A3 rs 1983023 were related with lower levels of TSH; however, the association did not reach statistical significance.

In conclusion; there was a significant correlation between rs225014 and rs225015 in DIO2 and TSH levels. However, DIO1, DIO3, UGT1A, THR a and TSHR were not associated L-T4 treatment. It should be lower dose in the individuals with DIO2 rs225014 (TT) and rs225015 $(G G)$ in order to provide the more effectively treatment with lower toxicity. The observed significant correlations should be sensitive and precious biomarkers of thyroid function and treatment among patients. Therefore, personalised medicine should be tendered optimal treatment according to patients' genetic background.

\section{Acknowledgements}

This work was supported by Istanbul University (BAP53871).

\section{References}

1. Al-Azzam SI, Alzoubi KH, Khabour O, Al-Azzeh O (2014) The associations of polymorphisms of TSH receptor and thyroid hormone receptor genes with L-thyroxine treatment in hypothyroid patients. Hormones (Athens) 13: 389-397.

2. Peeters RP, van der Deure WM, Visser TJ (2006) Genetic variation in thyroid hormone pathway genes; polymorphisms in the TSH receptor and the iodothyronine deiodinases. Eur J Endocrinol 155: 655-662.

3. Vanderpump MP (2011) The epidemiology of thyroid dis- ease. Br Med Bull 99: 39.

4. Wass JAH, Stewart PM (2011) Oxford Textbook of Endocrinology and Diabetes. 2nd Edition. Oxford University Press. ISBN: 978-0-19-923529-2.

5. Baskin HJ, Cobin RH, Duick DS, Gharib H, Guttler RB, et al. (2002) American Association of Clinical Endocrinologists medical guidelines for clinical practice for the evaluation and treatment of hyperthyroidism and hypothyroidism. Endocr Pract 8: 457-469.

6. Garber JR, Cobin RH, Gharib H, Hennessey JV, Klein I, 
et al. (2012) Clinical practice guidelines for hypothyroidism in adults: cosponsored by the American Association of Clinical Endocrinologists and the American Thyroid Association. Thyroid 22: 1200-1235.

7. Yardley L, Ainsworth B, Arden-Close E, Muller I (2015) The person-based approach to enhancing the acceptability and feasibility of interventions. Pilot Feasibility Stud 1: 37.

8. Al-Azzam SI, Alkhateeb AM, Al-Azzeh O, Alzoubi KH, Khabour OF (2013) The role of type II deiodinase polymorphisms in clinical management of hypothyroid patients treated with levothyroxine. Exp Clin Endocrinol Diabetes 121: 300-305.

9. Ciavardelli D, Bellomo M, Crescimanno C, Vella V (2014) Type 3 deiodinase: role in cancer growth, stemness, and metabolism. Front Endocrinol (Lausanne) 5; 215.

10. Santoro AB, Vargens DD, Barros Filho Mde C, Bulzico DA, Kowalski LP, et al. (2014) Effect of UGT1A1, UGT1A3, DIO1 and DIO2 polymorphisms on L-thyroxine doses required for TSH suppression in patients with differentiated thyroid cancer. Br J Clin Pharmacol 78: 10671075.

11. Wiersinga WM (2009) Do we need still more trials on T4 and T3 combination therapy in hypothyroidism? Eur $J$ Endocrinol 161: 955-959.

12. Den Hollander JG, Wulkan RW, Mantel MJ, Berghout A (2005) Correlation between severity of thyroid dysfunction and renal function. Clin Endocrinol (Oxf) 62: 423427.

13. Yoder Graber AL, Ramírez J, Innocenti F, Ratain MJ (2007) UGT1A1* 28 genotype affects the in-vitro glucuronidation of thyroxine in human livers. Pharmacogenet Genomics 17: 619-627.

14. Panicker V, Cluett C, Shields B, Murray A, Parnell KS, et al. (2008) A common variation in deiodinase 1 gene DIO1 is associated with the relative levels of free thyroxine and triiodothyronine. J Clin Endocrinol Metab 93: 3075-3081.
15. Torlontano M, Durante C, Torrente I, Crocetti U, Augello $\mathrm{G}$, et al. (2008) Type 2 deiodinase polymorphism (threonine 92 alanine) predicts L-thyroxine dose to achieve target thyrotropin levels in thyroidectomized patients. $J$ Clin Endocrinol Metab 93: 910-913.

16. Vargens DD, Neves RR, Bulzico DA, Ojopi ÉB, Meirelles RM, et al. (2011) Association of the UGT1A1-53 (TA) n polymorphism with L-thyroxine doses required for thyrotropin suppression in patients with differentiated thyroid cancer. Pharmacogenet Genomics 21: 341-343.

17. Panicker V, Saravanan P, Vaidya B, Evans J, Hattersley $\mathrm{AT}$, et al. (2009) Common variation in the DIO2 gene predicts baseline psychological well-being and response to combination thyroxine plus triiodothyronine therapy in hypothyroid patients. J Clin Endocrinol Metab 94: 16231629.

18. Hoftijzer HC, Heemstra KA, Visser TJ, le Cessie S, Peeters RP, et al. (2011) The type 2 deiodinase ORFaGly3Asp polymorphism (rs12885300) influences the set point of the hypothalamus-pituitary-thyroid axis in patients treated for differentiated thyroid carcinoma. $J$ Clin Endocrinol Metab 96: 1527-1533.

19. Heemstra KA, Hoftijzer HC, Van Der Deure WM, Peeters RP, Fliers E, et al. (2009) Thr92Ala polymorphism in the type 2 deiodinase is not associated with T4 dose in athyroid patients or patients with Hashimoto thyroiditis. Clin Endocrinol (Oxf) 71: 279-283.

20. Peeters RP, Van Toor H, Klootwijk W, De Rijke YB, Kuiper GG, et al. (2003) Polymorphisms in thyroid hormone pathway genes are associated with plasma TSH and iodothyronine levels in healthy subjects. J Clin Endocrinol Metab 88: 2880-2888.

21. van der Deure WM, Uitterlinden AG, Hofman A, Rivadeneira F, Pols HA, et al. (2008) Effects of serum TSH and FT4 levels and the TSHR-Asp727Glu polymorphism on bone: the Rotterdam Study. Clin Endocrinol (Oxf) 68: 175-181. 